\title{
Cardiac Troponin-I and COVID-19: A Prognostic Tool for In-Hospital Mortality
}

\author{
Baher AL Abbasi ${ }^{\mathrm{a}, \mathrm{d}}$, Pedro Torres ${ }^{\mathrm{a}}$, Fergie Ramos-Tuarez ${ }^{\mathrm{b}}$, Nakeya Dewaswala ${ }^{\mathrm{a}}$, Ahmed Abdallah ${ }^{\mathrm{a}}$, \\ Kai Chen ${ }^{\text {a }}$, Mohamed Abdul Qader ${ }^{a}$, Riya Job ${ }^{\text {a }}$, Samar Aboulenain ${ }^{\mathrm{a}}$, Karolina Dziadkowiec ${ }^{\mathrm{a}}$, \\ Huzefa Bhopalwala ${ }^{c}$, Jesus E. Pino ${ }^{\mathrm{b}}$, Robert D. Chait ${ }^{\mathrm{b}}$
}

\begin{abstract}
Background: The number of fatalities due to coronavirus disease 2019 (COVID-19) is escalating with more than 800,000 deaths globally. The scientific community remains in urgent need of prognostic tools to determine the probability of survival in patients with COVID-19 and to determine the need for hospitalization.
\end{abstract}

Methods: This is a retrospective cohort study of patients with a diagnosis of COVID-19 admitted to a tertiary center between March 2020 and July 2020. Patients age 18 years and older were stratified into two groups based on their troponin-I level in the first $24 \mathrm{~h}$ of admission (groups: elevated vs. normal). The aim of the study is to explore the utility of cardiac troponin-I level for early prognostication of patients with COVID-19.

Results: This cohort of 257 patients included 122/257 (47\%) women with a mean age of $63 \pm 17$ years. Patients with an elevated troponinI level were more likely to be older ( $77 \pm 13$ vs. $58 \pm 16$ years, $\mathrm{P}<$ $0.0001)$, have a history of hypertension $(\mathrm{P}<0.0001)$, diabetes mellitus $(\mathrm{P}=0.0019)$, atrial fibrillation or flutter $(\mathrm{P}=0.0009)$, coronary artery disease $(\mathrm{P}<0.0001)$, and chronic heart failure $(\mathrm{P}=0.0011)$. Patients with an elevated troponin-I level in the first $24 \mathrm{~h}$ of admission were more likely to have higher in-hospital mortality (52\% vs. $10 \%, \mathrm{P}<0.0001)$. Troponin-I level in the first $24 \mathrm{~h}$ of admission had a negative predictive value of $89.7 \%$ and a positive predictive value of $51.9 \%$ for all-cause in-hospital mortality.

Conclusions: Troponin-I elevation is commonly seen in patients with COVID-19 and is significantly associated with fatal outcomes. How-

Manuscript submitted September 2, 2020, accepted September 9, 2020

Published online October 23, 2020

aDepartment of Internal Medicine, University of Miami/JFK Medical Center Palm Beach Regional GME Consortium, Atlantis, FL, USA

${ }^{b}$ Department of Cardiology, University of Miami/JFK Medical Center Palm Beach Regional GME Consortium, Atlantis, FL, USA

'Department of Internal Medicine, Appalachian Regional Healthcare, Whitesburg, KY, USA

${ }^{\mathrm{d} C o r r e s p o n d i n g ~ A u t h o r: ~ B a h e r ~ A L ~ A b b a s i, ~ D e p a r t m e n t ~ o f ~ I n t e r n a l ~ M e d i c i n e, ~}$ University of Miami/JFK Medical Center, 5301 South Congress Ave, Atlantis, FL 33462, USA. Email: bsa56@med.miami.edu

doi: https://doi.org/10.14740/cr1159 ever, a normal troponin-I level in the first $24 \mathrm{~h}$ of admission had a high negative predictive value for all-cause in-hospital mortality, thereby predicting favorable survival at the time of discharge.

Keywords: SARS-CoV-2; COVID-19; Mortality; Troponin-I; Myocardial injury; Cardiovascular medicine

\section{Introduction}

Coronavirus disease 2019 (COVID-19) is a newly recognized infectious disease that has spread rapidly throughout most of the countries around the world. As of September 2020, more than 25 million laboratory-confirmed cases have been documented globally with more than 5 million cases in the USA. Furthermore, the number of fatalities due to COVID-19 continues escalating with more than 800,000 deaths globally thus far [1].

Cardiac troponin-I elevation has been historically known to predict worse mortality in both cardiovascular and noncardiovascular diseases [2-4]. With the increasing number of confirmed cases in the world, it has been recognized that the cardiovascular system plays a pivotal role. Moreover, the presence of myocardial injury (characterized by elevated cardiac troponin-I) has been significantly associated with a higher rate of morbidity and mortality in this population [5].

There is a lack of data regarding the role of a normal troponin-I within the first $24 \mathrm{~h}$ of admission in detecting early clinically relevant outcomes in this population.

This study evaluated the utility of a troponin-I level at the

\section{Key Points}

- Increases in cardiac troponin-I, indicative of myocardial injury, are common and prognostic in COVID-19.

- Troponin-I elevation is an accurate predictor of mortality in hospitalized patients with COVID-19.

- A normal cardiac troponin-I level on admission has a very high negative predictive value for all-cause in-hospital mortality.

- A normal cardiac troponin-I level on admission is a very strong and independent indicator of hospital survival.

- Cardiac troponin-I may facilitate COVID-19 stage classification and risk-stratification. 
time of admission and its ability to demonstrate a strong negative predictive value of all-cause in-hospital mortality in patients with COVID-19.

\section{Materials and Methods}

\section{Study design and participants}

This single-center, retrospective, observational study was performed at JFK Medical Center, Atlantis, FL, USA. We retrospectively analyzed patients with COVID-19 between March 2020 and July 2020, diagnosed by nasopharyngeal swab using the reverse transcription-polymerase chain reaction (RT-PCR). Clinical information was collected on admission and during hospitalization by the study team. The Institutional Review Board (IRB) at JFK Medical Center approved this study. This study was conducted in compliance with the ethical standards of the responsible institution on human subjects as well as with the Helsinki Declaration.

Patients age 18 years and older were initially stratified into two groups based on their cardiac troponin-I level in the first $24 \mathrm{~h}$ of admission (elevated vs. normal). A normal cardiac troponin-I level was defined as less than $0.012 \mathrm{ng} / \mathrm{mL}$, which falls below the 99th percentile in the blood test based on our facility's laboratory data. An elevated troponin-I value was defined as $\geq 0.012 \mathrm{ng} / \mathrm{mL}$. Furthermore, patients were grouped according to whether they died (non-survivors group) or survived (survivors group) the index hospitalization.

\section{Inclusion criteria}

Patients age 18 years and older who were hospitalized with a confirmed diagnosis of COVID-19, and had a troponin-I level drawn in the first $24 \mathrm{~h}$ of admission.

\section{Exclusion criteria}

Patients with acute coronary artery syndrome based on the fourth universal definition of myocardial infarction were excluded from this analysis [6]. Patients in which a troponin-I level was not obtained in the first $24 \mathrm{~h}$ of admission were also excluded, as were asymptomatic or mild cases that did not require hospitalization.

\section{Data collection}

The electronic medical records of the patients were reviewed by the study team. Patient data including demographics, medical history, laboratory examinations, comorbidities, complications, treatment measures, and outcomes were collected and analyzed.

\section{Outcome}

The primary outcome was the utility of cardiac troponin-I level for early prognostication of patients with COVID-19 and allcause in-hospital mortality or it is surrogate: discharge to hospice.

\section{Statistical analyses}

Statistical analyses were performed using the JMP program Version 14.0.0 (SAS Institute, Cary, North Carolina). Continuous variables were expressed as means with standard deviation $( \pm \mathrm{SD})$. A comparison of means (baseline characteristics, and predictors) was done using analysis of variance (ANOVA) (F statistic). A comparison of categorical variables was done using a Chi-square test. Results were considered significant if $\mathrm{P}$ values $<0.05$. Regression models were used for the primary outcome to adjust for common cofounders.

\section{Results}

A total of 432 cases with confirmed COVID-19 were screened between March 2020 and July 2020 at a tertiary cardiovascular center. Figure 1 depicts the flowchart for participant selection. After excluding cases without available core medical information, 257 hospitalized patients with COVID-19 were included in the final analysis. This cohort of 257 patients included $122 / 257(47 \%)$ women with a mean age of $63 \pm 17$ years.

\section{Comparison of clinical characteristics between elevated troponin and normal troponin groups}

As demonstrated in Table 1, patients with an elevated troponin-I level were older $(77 \pm 13$ vs. $58 \pm 16$ years, $\mathrm{P}<0.0001)$, have a history of hypertension $(\mathrm{P}<0.0001)$, diabetes mellitus $(\mathrm{P}=0.0019)$, atrial fibrillation or flutter $(\mathrm{P}=0.0009)$, coronary artery disease $(\mathrm{P}<0.0001)$, and chronic heart failure $(\mathrm{P}=$ 0.0011 ) compared to the normal troponin group. Furthermore, patients with an elevated troponin-I level on admission were more likely to have additional biochemical abnormalities at the time of admission, such as elevated blood urea nitrogen $(\mathrm{P}<0.0001)$, creatinine $(\mathrm{P}<0.0001)$, lactic acid $(\mathrm{P}=0.0046)$, serum sodium $(\mathrm{P}=0.0235)$, random blood sugar $(\mathrm{P}=0.0091)$, total bilirubin $(\mathrm{P}=0.0055)$, but a lower albumin level $(\mathrm{P}<$ $0.0001)$ compared to the normal troponin group.

\section{Comparison of clinical characteristics between non-survi- vors and survivors groups}

As demonstrated in Table 2, non-survivors (died during the index hospitalization or discharged to hospice) were more likely to be older $(\mathrm{P}<0.0001)$ and have a history of atrial fibrillation or flutter $(\mathrm{P}=0.0009)$. Non-survivors were also more likely to have a higher blood urea nitrogen $(\mathrm{P}<0.0001)$, creatinine $(\mathrm{P}=0.0097)$, random blood glucose $(\mathrm{P}=0.0026)$, lactic acid $(\mathrm{P}=0.0035)$, troponin-I $(\mathrm{P}<0.0001)$ and total bilirubin level $(\mathrm{P}=0.0045)$, but a lower albumin level $(\mathrm{P}=0.0004)$ compared to survivors. 


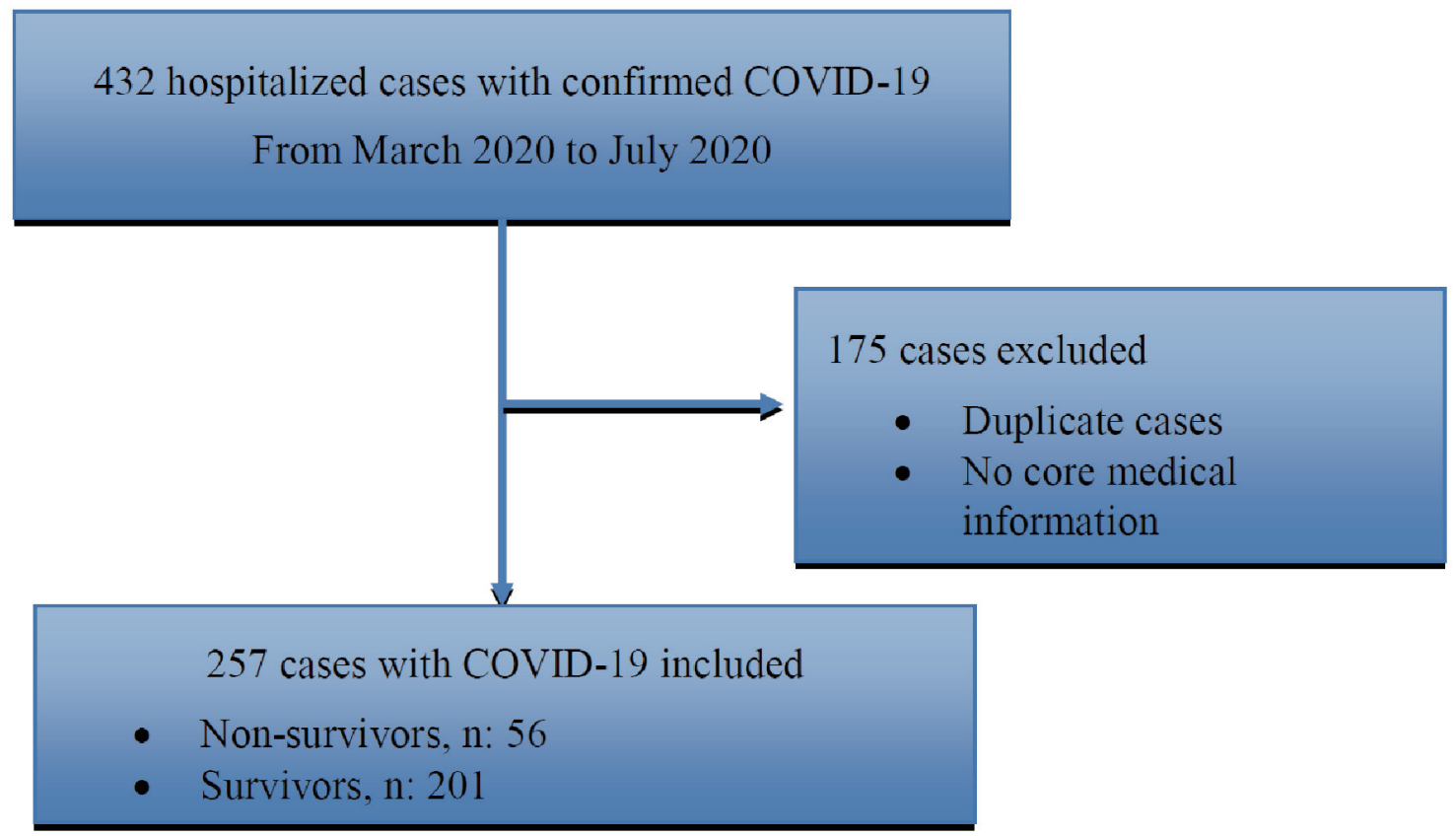

Figure 1. Flowchart for participants' selection. COVID-19: coronavirus disease 2019.

\section{Outcome}

In-hospital mortality or discharge to hospice occurred in 56/257 (21.7\%) patients diagnosed with COVID-19. Patients with an elevated troponin-I in the first $24 \mathrm{~h}$ of admission were more likely to have higher in-hospital mortality (52\% vs. $10 \%$, $\mathrm{P}<0.0001)$.

\section{Predictive value of cardiac troponin-I for in-hospital mortality}

As demonstrated in Table $3[7,8]$, a normal troponin-I level in the first $24 \mathrm{~h}$ of admission had a negative predictive value of $89.7 \%$ with a $95 \%$ confidence interval (CI) $(85.8 \%-92.7 \%)$ and a positive predictive value of $51.9 \%$ with $95 \%$ CI $(43 \%$ - $60.7 \%$ ) for all-cause in-hospital mortality (i.e., $89.7 \%$ is the probability that a patient with a normal cardiac troponin-I level on admission discharged home alive and $51.9 \%$ is the probability that a patient with an elevated cardiac troponin-I level on admission died during the index hospitalization).

A multivariate logistic regression analysis using Wald test was done to evaluate for possible confounders (age, atrial fibrillation, coronary artery disease, hypertension, diabetes mellitus, peripheral vascular disease, severe valvular disease, asthma, serum creatinine, lactic acid, total bilirubin, serum glucose, blood urea nitrogen, serum sodium, and absolute lymphocyte count). The logistic regression demonstrated that advanced age $(\mathrm{P}=0.0405)$, history of hypertension $(\mathrm{P}=0.0360)$, elevated troponin-I $(\mathrm{P}=0.0109)$, serum lactic acid $(\mathrm{P}=0.0264)$ and serum glucose $(P=0.0137)$ continued to be significant independent predictors of in-hospital mortality. This indicates that an elevated cardiac troponin-I remains an important and in- dependent predictor of mortality in patients with COVID-19.

\section{Discussion}

There is substantial evidence implicating COVID-19 as a cause of myocardial injury reflected by elevated cardiac troponin-I level [9-11]. This study demonstrates that patients with an elevated cardiac troponin-I level in the first $24 \mathrm{~h}$ of admission have a significantly higher in-hospital mortality when compared to patients with a normal troponin-I level. There are several hypotheses for COVID-19-associated myocardial injury, represented by troponin-I elevation, that are consistent with previous observations related to the outbreaks of severe acute respiratory syndrome (SARS) and the Middle East respiratory syndrome (MERS). Such mechanisms include myocarditis, microangiopathy, myocardial infarction, and cytokine storm [12].

Myocarditis is an inflammatory disease of the myocardium diagnosed by histological, immunological, and immunohistochemical criteria [13]. In COVID-19, particular attention has been given to the role of angiotensin-converting enzyme 2 (ACE2), the binding receptor for severe acute respiratory syndrome coronavirus 2 (SARS-CoV-2) cellular entry [14]. ACE2 is highly expressed in the pericytes of the heart, which suggests an intrinsic susceptibility of the heart to SARS-CoV-2 infection [15]. To date, only six known case reports of COVID-19 myocarditis in adults have been published, and three of them [16-18] have not been definitively proven by myocardial biopsy or cardiovascular magnetic resonance imaging. In the other three cases, [19-21], cardiovascular magnetic resonance scanning showed findings suggestive of myocarditis. This suggests that myocarditis is possible but less commonly the reason 
Table 1. Baseline Characteristics and Mortality of Patients with COVID-19 Based on the Cardiac Troponin-I level ( $\mathrm{N}=257)$

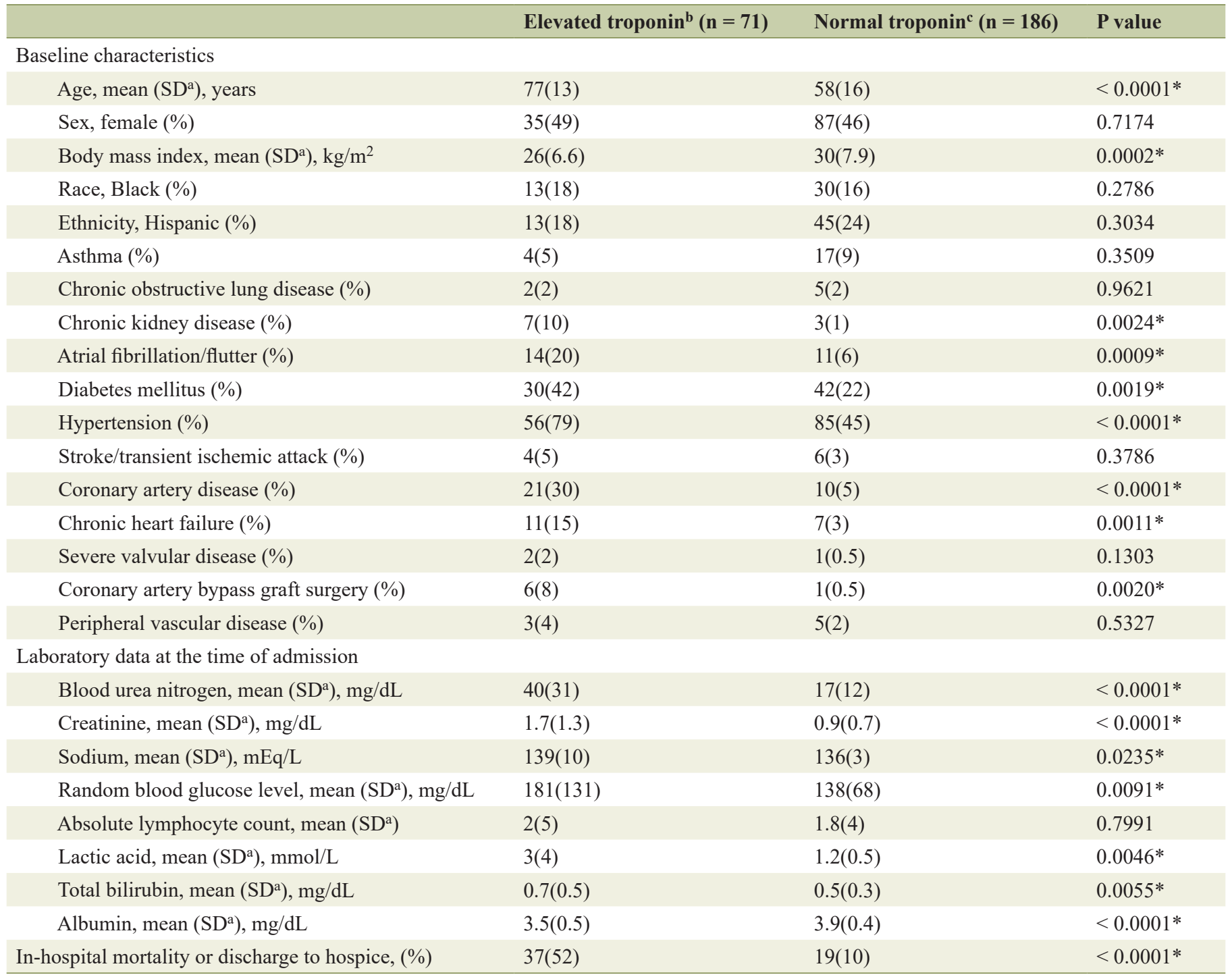

*Statistically significant. ${ }^{a} \pm$ SD. ${ }^{b}$ Elevated troponin: $\geq 0.012 \mathrm{ng} / \mathrm{mL}$ which falls above the 99th percentile in the blood test based on our facility's laboratory data. 'Normal troponin: $<0.012 \mathrm{ng} / \mathrm{mL}$ which falls below the 99th percentile in the blood test based on our facility's laboratory data. COVID-19: coronavirus disease 2019; SD: standard deviation.

for myocardial injury in this population.

Microangiopathy involves the capillaries, wherein the capillary walls become thickened and weak which results in bleeding, leakage of proteins, and disruption of blood flow. Endothelial dysfunction, oxidative stress, and angiotensin-II upregulation may explain the coagulopathy and microangiopathy frequently seen in severe coronavirus disease [22]. Oftentimes microangiopathy can precipitate thrombotic coagulopathy resulting in myocardial injury. Moreover, coagulopathy associated with SARS-CoV-2 has been widely reported [23]. Several studies have described an association between disease severity and D-dimer elevation, suggesting a thromboembolic tendency in patients with COVID-19 [24]. Additionally, some medical centers around the globe are using investigational therapeutic anticoagulation in patients with COVID-19 and elevated D-Dimer levels.
Myocardial infarction is one of the suggested hypotheses explaining troponin-I elevation. Patients with pre-existing coronary artery disease and those with risk factors for atherosclerotic cardiovascular disease are at an increased risk of developing acute coronary syndrome (ACS) during acute infections [25]. Troponin-I elevation could also result from an imbalance between oxygen supply and demand in the acute setting such that the troponin-I elevation may be interpreted as a type II myocardial infarction [26].

The occurrence of ACS in infected patients during the first SARS outbreak was also reported [27]. However, there is a paucity of data regarding ACS in patients with COVID-19. Based on the fourth universal definition of myocardial infarction [6], we excluded patients with ACS (ST-segment elevation myocardial infarction (STEMI) or patients with clinical 
Table 2. Comparison of Clinical Characteristics Between Non-Survivors and Survivors Groups $(N=257)$

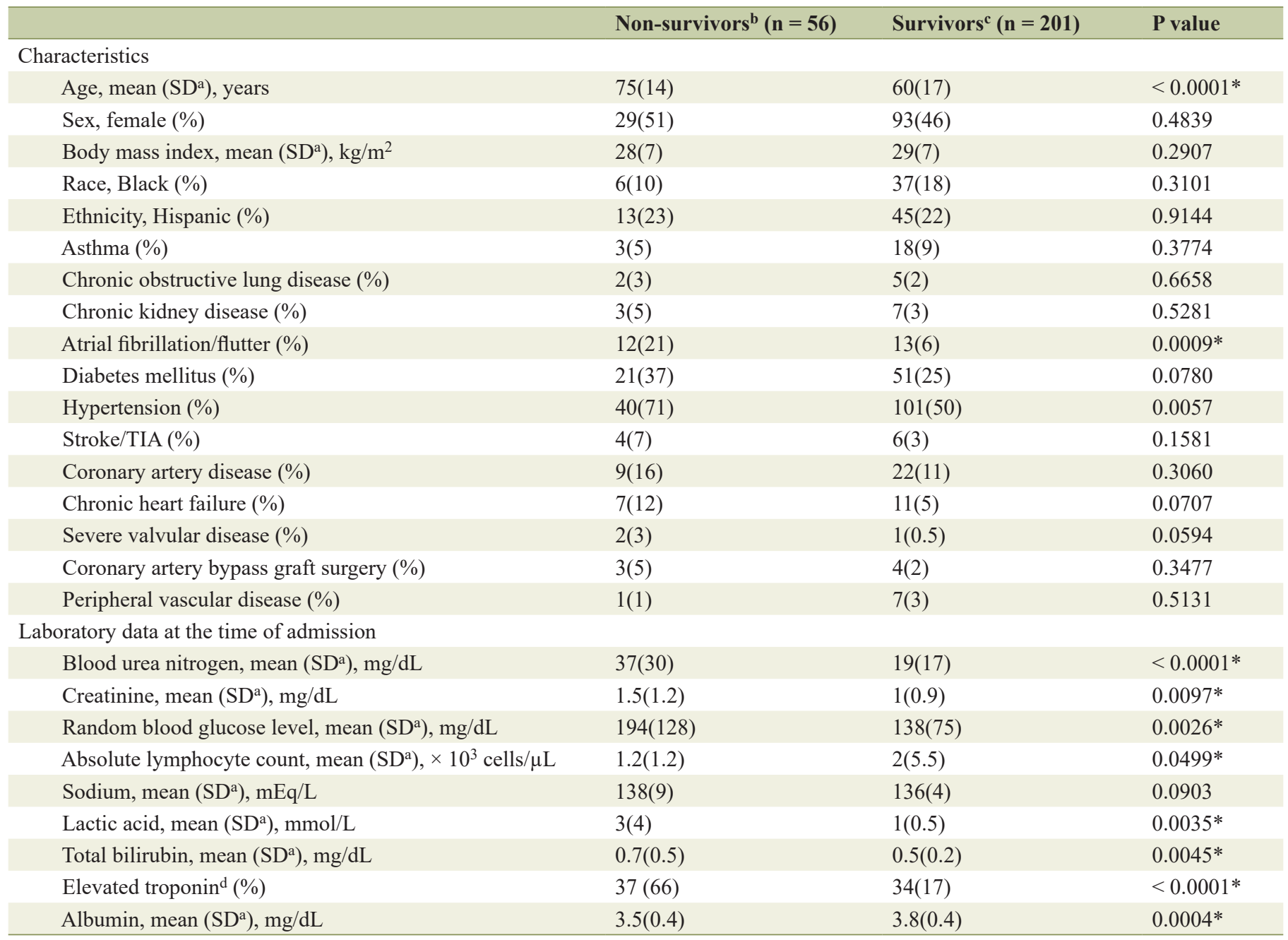

*Statistically significant. ${ }^{a} \pm$ SD. ${ }^{b}$ Non-survivors: patients died during the index hospitalization or discharged to hospice. ${ }^{c}$ Survivors: Patients were discharged home alive. 'Elevated troponin: $\geq 0.012 \mathrm{ng} / \mathrm{ml}$ which falls above the 99th percentile in the blood test based on our facility's laboratory data. SD: standard deviation; TIA: transient ischemic attack.

Table 3. Cardiac Troponin-I Level as a Predictor of Mortality in Patients with COVID-19

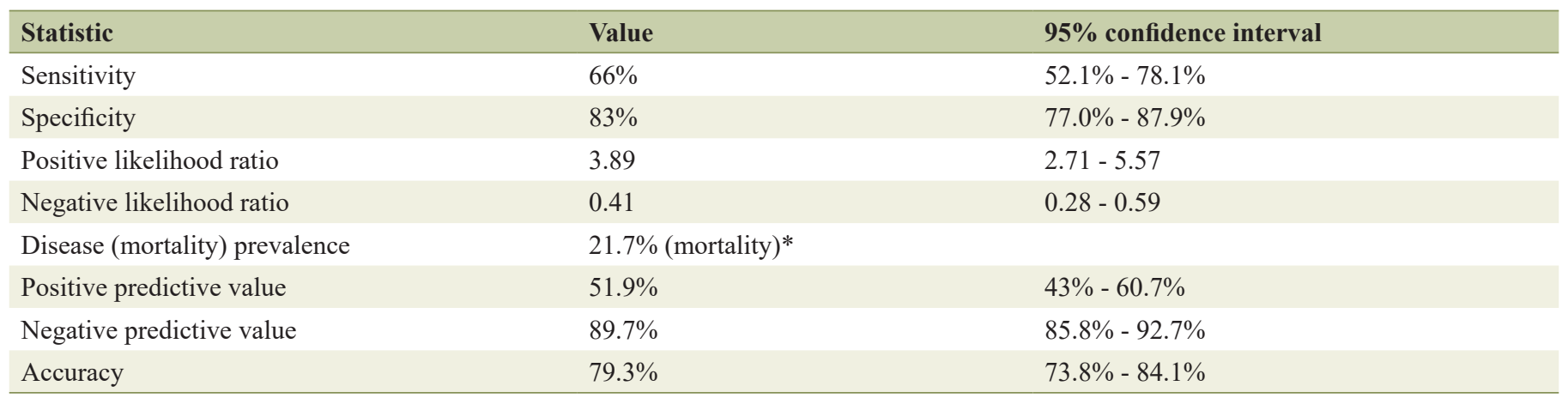

*The prevalence of all-cause in-hospital mortality for the study population. Sensitivity, specificity, disease (mortality) prevalence, positive and negative predictive value as well as accuracy are expressed as percentages. Confidence intervals for sensitivity, specificity and accuracy are "exact" ClopperPearson confidence intervals. Confidence intervals for the likelihood ratios are calculated using the "Log method" as given on page 109 of Altman et al 2000 [7]. Confidence intervals for the predictive values are the standard logit confidence intervals given by Mercaldo et al 2007 [8]. COVID-19: coronavirus disease 2019 . 
evidence of non-STEMI (NSTEMI) from the study population.

Cytokine storm has been extensively studied in patients with heart failure due to its role in infection, inflammatory modulation, myocyte stress, myocyte injury, fibroblast activation, and extracellular matrix remodeling [28]. C-reactive protein is one of the markers of cytokine storm and has been reportedly elevated in patients with COVID-19 and associated with higher mortality. Multiple new treatment modalities are being investigated to halt the effects of cytokine storm in patients with COVID-19 [29].

Given the myriad number of potential causes of troponin elevation, perhaps more attention should be focused on the advantage of a normal troponin level. As this study demonstrates, a normal troponin level in the first $24 \mathrm{~h}$ of admission has a high negative predictive value $(89.7 \%)$ for in-hospital mortality. Such a powerful biomarker can aid in risk stratifying patients at the time of admission and may allow for the implementation of more advanced therapies in those with higher risk. Conversely, a normal troponin-I value may allow physicians to identify those at lower risk and expedite discharge to preserve limited resources.

This study suggests that a single point of care cardiac troponin-I should be obtained in the first $24 \mathrm{~h}$ of admission in patients with COVID-19 regardless of the presence or absence of symptoms or other comorbidities.

In summation, troponin-I elevation is commonly seen in patients with COVID-19 and is significantly associated with fatal outcomes. Several mechanisms may explain this phenomenon: viral myocarditis, cytokine-driven myocardial damage, microangiopathy, ACS, and type II myocardial infarction. However, a normal troponin-I level on admission has a high negative predictive value to risk-stratify patients with $\mathrm{COV}$ ID-19. This suggests that troponin-I elevation is not only an accurate predictor of mortality, but its absence is also a very strong indicator of a more benign outcome.

\section{Limitations}

Similar to other observational studies of this nature several biases can be introduced. To control for this, a multivariable logistic regression was performed to evaluate for possible cofounders. Secondly, the patient population consists of hospitalized patients and does not represent asymptomatic or mildly symptomatic patients, in whom laboratory analysis is less likely to be performed. Finally, the mean age for this study population was $63 \pm 17$ years. This age group may underrepresent the younger patient population as they are more likely to be asymptomatic or have less severe disease not requiring hospitalization.

\section{Conclusions}

While troponin-I levels are commonly obtained to evaluate cardiac involvement in patients with COVID-19 and its elevation is associated with a higher all-cause in-hospital mortality, this study demonstrates that a normal troponin-I level, regardless cardiac involvement or other comorbidities, is a very strong indicator of hospital survival.

\section{Acknowledgments}

This research was supported (in whole or in part) by HCA Healthcare and/or an HCA Healthcare affiliated entity. The views expressed in this publication represent those of the author(s) and do not necessarily represent the official views of HCA Healthcare or any of its affiliated entities.

\section{Financial Disclosure}

None to declare.

\section{Conflict of Interest}

There are no relationships with any industry. All authors have reported that they have no relationships relevant to the contents of this paper to disclose.

\section{Informed Consent}

Not applicable.

\section{Author Contributions}

Baher AL Abbasi: study design and concept, data acquisition, data analysis and interpretation, and preparation of the first and subsequent manuscript drafts; Pedro Torres: study design and concept, data acquisition, and manuscript preparation and review; Fergie Ramos-Tuarez: manuscript review and editing; Nakeya Dewaswala: study design and concept, and manuscript review; Ahmed Abdallah: data acquisition and manuscript review; Kai Chen: data acquisition and manuscript review; Mohamed Abdul Qader: data acquisition and manuscript review; Riya Job: data acquisition and manuscript review; Samar Aboulenain: data acquisition and manuscript review; Karolina Dziadkowiec: data acquisition and manuscript review; Huzefa Bhopalwala: manuscript review; Jesus E. Pino: study design and concept, data analysis and interpretation, and manuscript review; Robert D. Chait: study design and concept, manuscript review and editing, and approving the final version.

\section{Data Availability}

The authors declare that data supporting the findings of this study are available within the article.

\section{Abbreviations}

COVID-19: coronavirus disease 2019; SARS-CoV-2: severe acute respiratory syndrome coronavirus 2 


\section{References}

1. COVID-19 Dashboard by the Center for Systems Science and Engineering (CSSE) at Johns Hopkins University (JHU). Available from: https://gisanddata.maps.arcgis.com/apps/ opsdashboard/index.html\#/bda7594740fd40299423467b 48e9ecf6?utm_source $=$ sn\&utm_medium $=$ referral\&utm 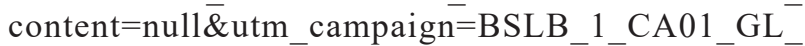 BSLB_AWA_CA01_GL_LSGR_PubH_Coronovirus_ LandingPage.

2. Antman EM, Tanasijevic MJ, Thompson B, Schactman $\mathrm{M}$, McCabe CH, Cannon CP, Fischer GA, et al. Cardiacspecific troponin I levels to predict the risk of mortality in patients with acute coronary syndromes. N Engl J Med. 1996;335(18):1342-1349.

3. Lorson W, Veve MP, Heidel E, Shorman MA. Elevated troponin level as a predictor of inpatient mortality in patients with infective endocarditis in the Southeast United States. BMC Infect Dis. 2020;20(1):24.

4. Vallabhajosyula S, Sakhuja A, Geske JB, Kumar M, Poterucha JT, Kashyap R, Kashani K, et al. Role of admission troponin- $\mathrm{T}$ and serial troponin- $\mathrm{T}$ testing in predicting outcomes in severe sepsis and septic shock. J Am Heart Assoc. 2017;6(9).

5. Sandoval Y, Januzzi JL, Jr., Jaffe AS. Cardiac Troponin for Assessment of Myocardial Injury in COVID-19: JACC Review Topic of the Week. J Am Coll Cardiol. 2020;76(10):1244-1258.

6. Thygesen K, Alpert JS, Jaffe AS, Chaitman BR, Bax JJ, Morrow DA, White HD, et al. Fourth universal definition of myocardial infarction (2018). J Am Coll Cardiol. 2018;72(18):2231-2264.

7. Altman DG, Machin David Bryant, Gardner TN, Martin J. Statistics with Confidence: Confidence Intervals and Statistical Guidelines, 2nd Edition. BMJ Books; 2000. p. 254.

8. Mercaldo ND, Lau KF, Zhou XH. Confidence intervals for predictive values with an emphasis to case-control studies. Stat Med. 2007;26(10):2170-2183.

9. Babapoor-Farrokhran S, Gill D, Walker J, Rasekhi RT, Bozorgnia B, Amanullah A. Myocardial injury and COVID-19: Possible mechanisms. Life Sci. 2020;253:117723.

10. Shi S, Qin M, Cai Y, Liu T, Shen B, Yang F, Cao S, et al. Characteristics and clinical significance of myocardial injury in patients with severe coronavirus disease 2019. Eur Heart J. 2020;41(22):2070-2079.

11. Rali AS, Ranka S, Shah Z, Sauer AJ. Mechanisms of myocardial injury in coronavirus disease 2019. Card Fail Rev. 2020;6:e15.

12. Tersalvi G, Vicenzi M, Calabretta D, Biasco L, Pedrazzini G, Winterton D. Elevated troponin in patients with coronavirus disease 2019: possible mechanisms. J Card Fail. 2020;26(6):470-475.

13. Caforio AL, Pankuweit S, Arbustini E, Basso C, GimenoBlanes J, Felix SB, Fu M, et al. Current state of knowledge on aetiology, diagnosis, management, and therapy of myocarditis: a position statement of the European Society of Cardiology Working Group on Myocardial and Pericardial Diseases. Eur Heart J. 2013;34(33):2636-2648, 2648a-2648d.

14. Hoffmann M, Kleine-Weber H, Schroeder S, Kruger N, Herrler T, Erichsen S, Schiergens TS, et al. SARS-CoV-2 Cell Entry Depends on ACE2 and TMPRSS2 and Is Blocked by a Clinically Proven Protease Inhibitor. Cell. 2020;181(2):271-280 e278.

15. Chen L, Li X, Chen M, Feng Y, Xiong C. The ACE2 expression in human heart indicates new potential mechanism of heart injury among patients infected with SARSCoV-2. Cardiovasc Res. 2020;116(6):1097-1100.

16. Inciardi RM, Lupi L, Zaccone G, Italia L, Raffo M, Tomasoni D, Cani DS, et al. Cardiac Involvement in a Patient With Coronavirus Disease 2019 (COVID-19). JAMA Cardiol. 2020;5(7):819-824.

17. Zeng JH, Liu YX, Yuan J, Wang FX, Wu WB, Li JX, Wang LF, et al. First case of COVID-19 complicated with fulminant myocarditis: a case report and insights. Infection. 2020;48(5):773-777.

18. Hu H, Ma F, Wei X, Fang Y. Coronavirus fulminant myocarditis saved with glucocorticoid and human immunoglobulin. Eur Heart J. 2020.

19. Doyen D, Moceri P, Ducreux D, Dellamonica J. Myocarditis in a patient with COVID-19: a cause of raised troponin and ECG changes. Lancet. 2020;395(10235):1516.

20. Kim IC, Kim JY, Kim HA, Han S. COVID-19-related myocarditis in a 21-year-old female patient. Eur Heart J. 2020;41(19):1859.

21. Besler MS, Arslan H. Acute myocarditis associated with COVID-19 infection. Am J Emerg Med. 2020.

22. Yin S, Huang M, Li D, Tang N. Difference of coagulation features between severe pneumonia induced by SARSCoV2 and non-SARS-CoV2. J Thromb Thrombolysis. 2020.

23. Jhaveri KD, Meir LR, Flores Chang BS, Parikh R, Wanchoo R, Barilla-LaBarca ML, Bijol V, et al. Thrombotic microangiopathy in a patient with COVID-19. Kidney Int. 2020;98(2):509-512.

24. Garcia-Olive I, Sintes H, Radua J, Abad Capa J, Rosell A. D-dimer in patients infected with COVID-19 and suspected pulmonary embolism. Respir Med. 2020;169:106023.

25. Corrales-Medina VF, Madjid M, Musher DM. Role of acute infection in triggering acute coronary syndromes. Lancet Infect Dis. 2010;10(2):83-92.

26. Thygesen K, et al. Fourth universal definition of myocardial infarction (2018). European Heart Journal. 2018;40(3):237-269.

27. Chong PY, Chui P, Ling AE, Franks TJ, Tai DY, Leo YS, Kaw GJ, et al. Analysis of deaths during the severe acute respiratory syndrome (SARS) epidemic in Singapore: challenges in determining a SARS diagnosis. Arch Pathol Lab Med. 2004;128(2):195-204.

28. Gullestad L, Ueland T, Vinge LE, Finsen A, Yndestad A, Aukrust P. Inflammatory cytokines in heart failure: mediators and markers. Cardiology. 2012;122(1):23-35.

29. Sinha P, Matthay MA, Calfee CS. Is a "Cytokine Storm" relevant to COVID-19? JAMA Intern Med. 2020. 